\title{
MFID: uma Solução Computacional para a Identificação da Fadiga Muscular Durante a Reabilitação Física de Indivíduos Amputados
}

\author{
Bryan Teixeira Paiva \\ Universidade Federal do Pampa - UNIPAMPA \\ bryanpaiva20@gmail.com \\ Jone Follmann \\ Universidade Federal do Pampa - UNIPAMPA \\ jone.follmann.ipo@gmail.com
}

\author{
Érico Marcelo Hoff do Amaral \\ Universidade Federal do Pampa - UNIPAMPA \\ ericoamaral@unipampa.edu.br \\ Julio Domingues Saraçol Júnior \\ Universidade Federal do Pampa - UNIPAMPA \\ juliodomingues@unipampa.edu.br
}

\begin{abstract}
It's know that computing is becoming increasingly integrated into other knowledge areas, providing practical and innovative solutions to many types of problems, including to health care area. In this sense, the amputatee patients physical rehabilitation process also shows as an important area for tools development that assist the professional physiotherapist, since this rehabilitation process is long-lasting and exhaustive for patients. Thus, the present work describes the development of a solution called Muscle Fatigue Identifier (MFID) to improve the amputee physical rehabilitation process, by detecting muscle fatigue during physical exercise sessions, which once unidentified, can cause injury and pain to patients, further prolonging treatment. The developed system consists in the integration of sensors, hardware and software. This way, it was possible to monitoring and identify the muscle fatigue occurrence during the physical exercise sessions performed by the patients, as well as allowing a use tool to evaluate the physiotherapy treatment.
\end{abstract}

\section{KEYWORDS}

FADIGA MUSCULAR, INDIVÍDUOS AMPUTADOS, MONITORAMENTO, REABILITAÇÃO FÍSICA

\section{INTRODUÇÃO}

A fadiga muscular é um dos fenômenos mais comuns encontrados por indivíduos que realizam algum tipo de exercício físico, e pode ser definida como a incapacidade de manter o rendimento durante o exercício em condições extremas de intensidade ou duração. Também tem como característica, a inabilidade do músculo em gerar elevados níveis de força muscular ou mantê-los durante um determinado tempo e o seu desencadear pode tanto causar lesões em pessoas que estão se iniciando na atividade física, quanto ocasionar grandes prejuízos para atletas. Além disso, na locomoção patológica, como de amputados e indivíduos com restrição neurológica, onde há alterações no padrão de movimento e no esforço físico, a instauração da fadiga pode prejudicar o desempenho durante o processo de reabilitação física dos pacientes [1].

Neste sentido, o processo de reabilitação física por meio da fisioterapia é uma importante ferramenta para o bem-estar físico e emocional do indivíduo. O fisioterapeuta desempenha um papel fundamental quanto à reeducação funcional, acompanhando o paciente em todos os estágios do programa de reabilitação. Assim, a presença do fisioterapeuta é importante no processo dinâmico, progressivo, educativo e, objetiva a restauração do indivíduo, sua reintegração à família, comunidade e sociedade [2].

Desse modo, vale ressaltar que a Informática Médica é uma das áreas que vem se destacando no cenário da computação com diversos avanços tecnológicos para a área da saúde, desempenhando um papel importante na coleta, análise, processamento e armazenamento de dados. Levando em consideração o âmbito da fisioterapia, existe uma enorme gama de oportunidades para o desenvolvimento de tecnologias que auxiliem o profissional fisioterapeuta durante as diversas etapas do processo de reabilitação física de pacientes, tornando o tratamento mais eficiente e o diagnóstico mais preciso.

Considerando o contexto apresentado, o objetivo desta pesquisa é a identificação da fadiga muscular durante as sessões de exercícios físicos para evitar lesões e agravamento no estado físico de pacientes, além de propiciar ao fisioterapeuta uma ferramenta para o tratamento adequado às necessidades específicas de cada paciente.

Assim sendo, além desta seção de introdução, este documento apresenta as seguintes seções: a metodologia empregada na seção 2; o referencial teórico levantado na seção 3; na seção 4 o projeto MFID; o desenvolvimento da pesquisa na seção 5 ; os experimentos e validação da ferramenta na seção 6; na seção 7 serão discutidos os resultados obtidos; por fim, na seção 8 são realizadas as considerações e conclusões sobre a pesquisa.

\section{METODOLOGIA}

Com o propósito de alcançar os objetivos de pesquisa, através de uma sequência de atividades bem definida e estruturada, foi estabelecida uma metodologia de pesquisa. Esta metodologia foi seguida durante todo o decorrer do trabalho, objetivando o desenvolvimento da solução de maneira coesa e organizada.

Quanto a sua natureza, a pesquisa pode ser classificada como aplicada, visto que objetiva gerar conhecimentos para aplicação prática e dirigidos à solução de problemas específicos. A forma de abordagem do problema é quantitativa, ou seja, representa, classifica e analisa na forma de números as informações obtidas. Além disso, a pesquisa também pode ser classificada como experimental, em razão de que esta determina um objeto de estudo, identifica as variáveis que são capaz de influenciá-lo e define as formas de observação dos efeitos que as variáveis produzem sobre o objeto [3].

Segundo [4], uma pesquisa científica é composta por sete etapas: a formulação da questão inicial; a exploração da questão inicial; a 
elaboração da problemática; a construção de um modelo de análise; a coleta de dados; a análise das informações e as conclusões. Assim sendo, a Figura 1 representa, em um diagrama de atividades, as etapas de desenvolvimento da presente pesquisa.

A primeira etapa da metodologia se consistiu na definição do problema de pesquisa. Esta definição iniciou-se a partir da análise de testes realizados pelo Grupo de Informática Médica (GIM) com pacientes do Serviço de Reabilitação Física (SRF) do município de Bagé-RS, onde foi evidenciada a ocorrência da fadiga muscular nos pacientes, como também foi observada a não existência de equipamentos para mensurar as condições físicas dos pacientes e identificar esse tipo de fenômeno. Dessa forma, foi constatada a necessidade de uma solução computacional para a identificação da fadiga muscular durante as sessões de fisioterapia, com o objetivo de aperfeiçoar o tratamento de reabilitação física.

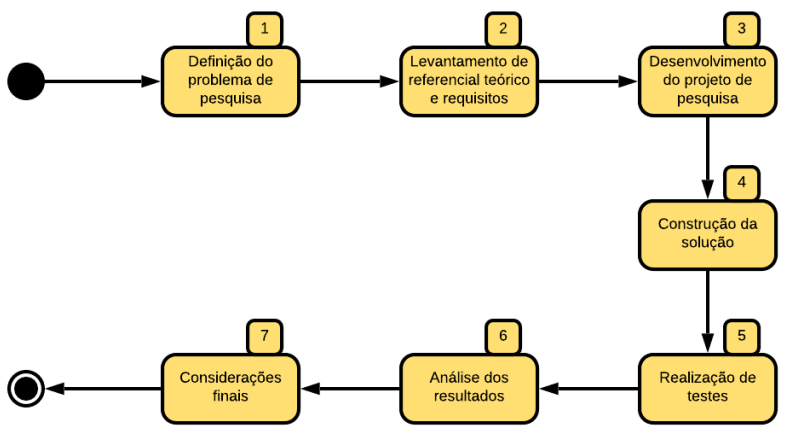

Figura 1: Metodologia representada em um diagrama de atividades.

Após definido o problema de pesquisa, foi necessário o levantamento de um referencial teórico para ser utilizado como base de estudos no desenvolvimento da pesquisa. Posteriormente, foi desenvolvido o projeto de pesquisa, para isto, se utilizou toda a base de conhecimento adquirida durante a etapa anterior, como o levantamento de referencial teórico e elicitação de requisitos.

Na quarta etapa da metodologia foi desenvolvido o sistema MFID, o qual seguiu as etapas previstas no projeto da etapa anterior. A ferramenta foi construída a partir da integração de sensores, hardware e software. Em seguida, a próxima etapa da metodologia realizada foi a de testes da solução desenvolvida. Inicialmente, foram realizados testes unitários dos componentes que compõem a solução, e após, foram realizados os testes de integração da solução. Por fim, foi definido o público-alvo, constituído por pacientes do SRF, para a realização dos testes de validação do sistema desenvolvido.

Na sexta etapa, com o intuito de avaliar a funcionalidade e desempenho da solução, foram realizadas análises e avaliações dos resultados obtidos durante a etapa de testes da ferramenta. Por fim, na última etapa da metodologia, foram realizadas análises e discussões sobre o desenvolvimento da pesquisa. Desse modo, foram abordados nesta análise, todos os pontos do trabalho, desde os métodos aplicados no desenvolvimento do projeto, até os resultados obtidos e o desempenho da solução construída.

\section{REFERENCIAL TEÓRICO}

Nesta seção de referencial teórico são apresentadas fundamentações teóricas relacionadas aos principais temas envolvidos no desenvolvimento deste trabalho. Assim, é relevante a absorção das informações contidas neste referencial para um melhor entendimento das bases de conhecimentos estudadas durante a pesquisa.

Primeiramente é abordada a fadiga muscular, bem como suas características, causas e consequências. Após, é apresentada a amputação e suas características gerais. Em seguida, são expostos os principais conceitos sobre o processo de reabilitação física. Por fim, é discutido a atuação e importância da informática médica no âmbito da saúde.

\subsection{Fadiga muscular}

Nas atividades diárias do cotidiano, no trabalho, no esporte e em qualquer atividade física realizada, existe a presença da fadiga muscular, apresentando-se muitas vezes como um limitante do desempenho humano e causadora de lesões em diversos níveis do sistema músculo-esquelético [5]. É frequentemente caracterizada pela falta de energia, sensação de cansaço e exaustão, se relacionando com a dificuldade na realização de tarefas voluntárias.

Uma recente área de estudos sobre a fadiga muscular têm sido realizado no âmbito da recuperação funcional de sujeitos com patologias neuromusculares ou lesões em determinadas estruturas do sistema nervoso. Alguns estudos relacionaram as alterações de parâmetros cinemáticos induzidas pela fadiga na identificação de fatores de risco para ocorrência de lesões de sobrecarga [6].

Neste contexto, de acordo com [7], em pacientes amputados, dependendo do tipo de amputação, a fadiga muscular tende a ser ainda mais presente, os amputados transfemorais apresentam significativa redução da força no membro amputado comparado ao não amputado. Amputados de membros inferiores não têm muitos mecanismos naturais que atenuam o impacto das forças durante a marcha, além disso, os tecidos moles do membro residual não são particularmente bem adaptados para tolerar a carga mecânica, o que pode levar à dor e lesões na pele [8].

No que diz respeito a identificação e monitoramento da fadiga muscular, atualmente são utilizadas distintas técnicas, sendo as principais e mais promissoras a eletromiografia e a mecanomiografia.

A eletromiografia (EMG) é essencialmente o registro elétrico da atividade da unidade motora. Esse registro possibilita, mediante a interpretação dos sinais elétricos, o estudo da ativação das fibras musculares presente em todo e qualquer movimento executado pela musculatura, permitindo o acompanhamento de variáveis associadas ao desempenho do músculo estudado, tais como força produzida e a velocidade de propagação do potencial de ação [9].

Nesse sentido, [9] definem a eletromiografia como um método de investigação do estado fisiológico do sistema neuromuscular, empregada muitas vezes na avaliação do alcance da doença neuromuscular e como instrumento cinesiológico para o estudo da função muscular. Seguindo este raciocínio, [10] indicam que através da eletromiografia pode-se analisar o comportamento de algumas patologias, prevenção de lesões musculares, análise de força, fadiga muscular, entre outros.

A mecanomiografia (MMG) é a técnica que registra as vibrações e oscilações do músculo que ocorrem quando este se contrai. Uma 
vibração mecânica na pele sobre um músculo em contração é produzida por mudanças dimensionais laterais das fibras musculares ativas [11]. Essas oscilações, e os sons correspondentes, podem ser detectados por meio de acelerômetros colocados sobre a superfície da pele, transformando a vibração em sinal elétrico. A MMG de superfície é o resultado da somatória dos sinais emitidos pelo número de unidades motoras ativas, mediadas e moduladas pela arquitetura do complexo músculo-tendão, gordura e pele.

De acordo com [12], a mecanomiografia pode ser útil para a investigação do músculo nas áreas da fisiologia, medicina clínica e reabilitação. Nesse contexto, clinicamente a MMG pode ser utilizada para examinar desordens neuromusculares, incluindo paralisia cerebral, distrofia miotônica, desordens craniomandibulares, dor lombar crônica e severa, fadiga muscular, atrofia muscular esquelética, e como um mecanismo de controle externo de próteses.

\subsection{Amputação}

De acordo com o Ministério da Saúde, amputação é o termo utilizado para definir a retirada total ou parcial, geralmente de forma cirúrgica, de um membro ou extremidade do corpo, sendo o membro residual da amputação denominado de coto. Vale ressaltar que o procedimento de amputação não pode ser visto unicamente como o propósito final da cirurgia, mas sim como parte de um processo maior que objetiva o tratamento de diversas doenças e assim prover uma melhora na qualidade de vida do paciente. Assim, a decisão de amputação, apesar de tudo, é encarada como um bem necessário para pôr fim ao sofrimento do paciente [13].

Ao realizar uma amputação, deve-se ter cuidadosa consideração à escolha do nível, de modo a deixar o coto com as maiores perspectivas de ser protetizado. Neste sentido, com o intuito de uma melhor qualidade de vida e adaptabilidade do paciente no processo de protetização, um nível de amputação será mais adequado do que outro quanto melhor se prestar a adaptação de uma prótese [14].

Segundo [13], a utilização de próteses tem como objetivo minimizar as incapacidades e desvantagens de quem teve um sofrimento profundo ao ser privado de um membro, proporcionando ao paciente a capacidade de ser independente e para o melhor e mais breve possível retorno às atividades habituais e assim, readquirir sua auto-estima, a vontade e a alegria de viver.

Assim sendo, logo após a amputação e durante a etapa de protetização de um paciente amputado, este necessita de tratamento de reabilitação física e de um acompanhamento profissional de um fisioterapeuta. O Ministério da Saúde destaca que o fortalecimento muscular do coto de amputação é extremamente importante, e enfatiza, também, que após a amputação o paciente utilizará outros grupos musculares para executar suas atividades de vida diária. Portanto, o fortalecimento muscular deve ser geral.

\subsection{Reabilitação Física}

Segundo a Organização Mundial da Saúde, o processo de reabilitação física pode ser definido como o uso de todos os meios necessários para reduzir o impacto da condição incapacitante e permitir aos indivíduos incapacitados a obtenção de uma completa integração. Assim, a reabilitação tem como objetivo, reduzir a incapacidade provocada por alguma doença e, quando possível, melhorar a capacidade funcional do indivíduo.
A reabilitação é um processo global e dinâmico que objetiva a reintegração social do paciente, recuperar a saúde física e psicológica da pessoa portadora de deficiência ou com funções prejudicadas por doença ou evento traumático. Entretanto, a reabilitação não pode ser considerada uma estratégia isolada, mas sim uma estratégia integrada ao tratamento do usuário [15].

Com a amputação o paciente sofre grande alteração funcional do músculo esquelético e dificuldades na adaptação a uma condição incapacitante, pois o mesmo necessita fazer mudanças na sua vida, desde sociais, econômicas e até familiares [16]. Entretanto, além dos fatores físicos associados à amputação, também existem os fatores psicológicos relacionados à ela, [13] ressalta que a amputação pode ocasionar distúrbios emocionais como a perda da identidade, baixa auto estima, sentimento de inferioridade e depressão. Fatores estes que podem desmotivar e interferir negativamente no processo de reabilitação física do paciente.

Nesse contexto, [17] aponta que o fisioterapeuta desempenha papel fundamental quanto à reeducação funcional, acompanhando o paciente em todos os estágios do programa de reabilitação, supervisionando e tratando desde o estágio pré e pós-operatório, na educação de mobilidade pré e pós-protética e, se necessário, em cuidados de manutenção das funções músculo-esqueléticas.

Contudo, mesmo sendo um serviço importante para o bem-estar físico, psicológico e social do paciente, o resultado de qualquer programa de reabilitação, inclusive de pacientes amputados, é dependente da participação ativa do paciente, que por sua vez é muito influenciada pela atitude e motivação do indivíduo para a abordagem de reabilitação física a qual ele está sendo exposto [18].

Nesta perspectiva, é evidente a necessidade do desenvolvimento de novos métodos e ferramentas tecnológicas para uma maior eficácia no processo de reabilitação física e tornar o tratamento mais motivador. Sendo assim, a informática médica é a área de estudo responsável pelo desenvolvimento e aplicação de técnicas para melhorar este panorama.

\subsection{Informática Médica}

Segundo [19], a informática médica é o estudo e a aplicação de métodos para melhorar o gerenciamento de dados de pacientes, conhecimento clínico, dados populacionais e outras informações relevantes para o cuidado do paciente e a saúde da comunidade. Também pode ser definida como o campo científico que lida com informações, dados e conhecimentos biomédicos para resolução de problemas e tomada de decisões. Por conseguinte, aborda todos os campos básicos e aplicados da ciência biomédica e é estreitamente ligados às modernas tecnologias da informação.

O estudo da informática tem sido cada vez mais fundamental para as práticas da medicina e da prestação de cuidados de saúde. Entretanto, ao invés de novos medicamentos, máquinas de raios-x, ou novos instrumentos cirúrgicos, as ferramentas computacionais são mais propensas a serem utilizadas, como sistemas de apoio à tomada de decisão, registros eletrônicos ou sistemas de comunicação [20]. Assim, aprimorando o uso da informação e tornando mais eficiente a forma como os dados do paciente e o conhecimento médico são capturados, processados, comunicados e aplicados.

Considerando que os profissionais da área de saúde devam desenvolver competências e saberes tecnológicos que possam enriquecer e possibilitar a construção de novos conhecimentos e instrumentos 
que estejam voltados para a sua prática profissional, torna-se cada vez mais imprescindível a discussão e apresentação de propostas que objetivem a utilização de recursos computacionais na área da saúde. Contudo, [21] ressalta que nenhum sistema pode ou deve substituir o olhar do profissional ao examinar o paciente, buscando todos os sinais e sintomas que venham a tornar possível o diagnóstico. Porém, o uso de sistemas especialistas que venham auxiliar na sistematização do processo de tomada de decisões podem contribuir de forma significativa com o profissional na sua prática.

Uma das grandes vantagens de um sistema especialista está no fato deste não ser influenciado por elementos externos a ele, como ocorre com o especialista humano [22]. Para as mesmas condições, o sistema deverá fornecer sempre o mesmo conjunto de decisões, isto elimina erros devido à pressão do ambiente, ou seja, situações críticas que necessitam de respostas rápidas e precisas.

\section{PROJETO MFID}

A partir da constatação da necessidade de desenvolver uma solução computacional para a identificação da fadiga muscular durante as sessões de reabilitação física, foi então, iniciado este projeto, intitulado Muscle Fatigue Identifier ou, simplesmente, MFID. A ideia inicial deste projeto foi desenvolver uma solução simples, de baixo custo e que atue em conjunto com o fisioterapeuta na análise e monitoramento da fadiga muscular durante o processo de reabilitação física de pacientes amputados.

Deste modo, com base nos conceitos discutidos na seção de referencial teórico, chegou-se à conclusão de que, para evitar lesões de pacientes, se fez necessário uma solução capaz de identificar a fadiga muscular no início de seu surgimento e apresentar, em tempo de execução, essa informação ao fisioterapeuta. Para que o dados fossem apresentados de forma clara ao fisioterapeuta, um dos requisitos para a construção deste projeto foi o desenvolvimento de uma interface gráfica passível de apresentar um conjunto de indicadores relevantes sobre a fadiga muscular.

Analisando mais especificamente alguns aspectos apresentados na Seção 3.1, definiu-se que a solução abordaria o problema de pesquisa a partir de um sistema microcontrolado e empregando duas técnicas de detecção de fadiga muscular, a eletromiografia e a mecanomiografia. Para isto, foram utilizados sensores específicos para a coleta dos dois sinais e um microcontrolador para o processamento dos sinais, além de um software para a visualização e armazenamento dos dados. Assim sendo, a Figura 2 apresenta de forma ilustrativa a ideia conceitual do projeto.

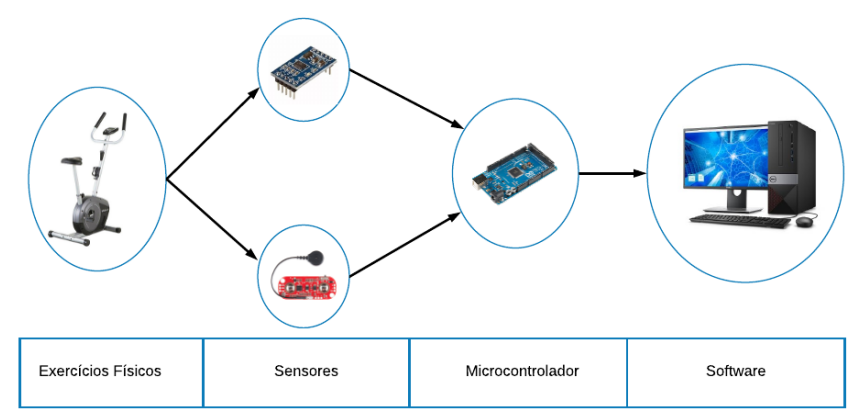

Figura 2: Modelo conceitual - Sistema MFID.
Em resumo, o projeto MFID consiste em mensurar e apresentar, graficamente, as informações sobre fadiga muscular coletadas através de sensores posicionados no músculo de pacientes amputados durante as sessões de fisioterapia, as quais consistem na execução de um conjunto de exercícios, seguindo um protocolo específico. Através dos dados apresentados pelo MFID, o fisioterapeuta poderá identificar a instauração da fadiga durante a realização da atividade e, a partir disso, tomar os devidos cuidados para evitar lesões e desgastes musculares dos pacientes, podendo reduzir a carga de exercício ou até mesmo interromper a sessão.

\section{DESENVOLVIMENTO}

Para uma melhor organização de trabalho, o desenvolvimento da solução foi divida em duas etapas, a construção do nó sensor e a implementação do software. Desta maneira, esta seção apresenta as definições de projeto das duas etapas de desenvolvimento da solução.

\subsection{Nó sensor}

O nó sensor é parte do sistema responsável, a partir dos sensores e do microcontrolador, pela coleta e processamento dos sinais de EMG e MMG oriundos dos músculos. Desta forma, foram elencadas as seguintes tecnologias para a construção da solução: uma plataforma de prototipagem eletrônica Arduino Mega 2560, um sensor de EMG MyoWare Muscle Sensor, e um acelerômetro triaxial MMA7361.

A placa Arduino Mega 2560 é baseada no microcontrolador Atmel ATmega2560, este microcontrolador tem como principais características um processador RISC com até 16 MIPS, $256 \mathrm{kB}$ de memória Flash, 8 kB de memória SRAM e 4 kB de memória EEPROM. A escolha desta placa para o projeto foi, principalmente, por esta possuir uma capacidade de armazenamento satisfatória para o desenvolvimento da solução, além da grande quantidade de pinos de entrada e saída que oferece.

O sensor de eletromiografia MyoWare Muscle Sensor é capaz de mensurar, de forma não invasiva, a atividade muscular através da variação do seu potencial elétrico. Para flexionar o músculo, o cérebro envia um sinal elétrico para o músculo começar a recrutar unidades motoras e, quanto maior for a flexão, mais unidades motoras são recrutadas para gerar maior força muscular. Assim, o sensor de EMG analisa essa atividade elétrica, através do sinal de entrada de tensão bruta, e emite um sinal de saída analógico. Assim, este sinal representa o quanto o músculo está sendo flexionado.

O outro sensor utilizado nesta pesquisa é o sensor de vibração Acelerômetro MMA7361. Este sensor é capaz de medir a inclinação e aceleração tridimensional de um objeto, gerando uma variação de tensão, para cada eixo, correspondente a aceleração detectada.

Assim, após a coleta dos sinais de EMG e MMG pelos sensores, o próximo passo é o processamento destes sinais para analisar o comportamento de suas amplitudes e frequências. Desse modo, para a análise da amplitude foi utilizada como métrica uma medida de magnitude frequentemente mencionada e utilizada, tanto para a EMG quanto para a MMG, em estudos sobre fadiga muscular [12, 23-26], a RMS (do inglês, Root Mean Square).

Dessa maneira, a partir da análise da amplitude do sinal através da RMS é possível identificar diversas características do músculo, como a taxa de disparo e o recrutamento das unidades motoras 
ativas durante a contração, como também a força desenvolvida durante a atividade [27]. A Equação 1 apresenta o cálculo da RMS, onde $\mathrm{N}$ é o número de amostras consideradas, $\mathrm{m}$ é o índice da m-ésima amostra do segmento e $X_{m}$ é o valor da amostra.

$$
R M S=\sqrt{\frac{1}{N} \sum_{m=1}^{N} X_{m}^{2}}
$$

No domínio da frequência, a análise da frequência média (FM) do espectro de potência (PSD) tem sido utilizada por diferentes autores [11, 23-26, 28] no estudo da fadiga muscular. Assim, para a análise da frequência se utilizou uma biblioteca para Arduino a qual realiza a Transformada Rápida de Fourier (FFT).

A FM é calculada a partir da média aritmética dos valores de frequência obtidas no PSD. Dessa forma, a partir da análise através da frequência média e de suas mudanças no decorrer do tempo, é possível identificar algumas características do músculo, como velocidade de condução e ativação das fibras musculares [26, 28].

Desse modo, o nó sensor é capaz de coletar e processar os dados de EMG e MMG e, após isto, enviar esses dados, através da porta serial, para um computador onde uma aplicação, para a visualização gráfica e armazenamento das informações é executada. Assim sendo, a seguir é apresentado um detalhamento sobre o software desenvolvido neste projeto.

\subsection{Software}

Para implementar o módulo responsável pela apresentação das informações ao usuário, foi desenvolvida uma aplicação em linguagem de programação Java integrada a um banco de dados MySQL. Na aplicação, os dados de EMG e MMG são recebidos por meio da API de comunicação serial RXTX, a qual possibilita abrir a porta serial para a comunicação e realizar a leitura e manipulação dos dados enviados pelo Arduino. Desse modo, é possível gerar os gráficos e indicadores sobre a fadiga muscular a partir dos sinais coletados pelos sensores posicionados na musculatura de pacientes. Assim, a Figura 3 apresenta a tela de monitoramento do sistema MFID.

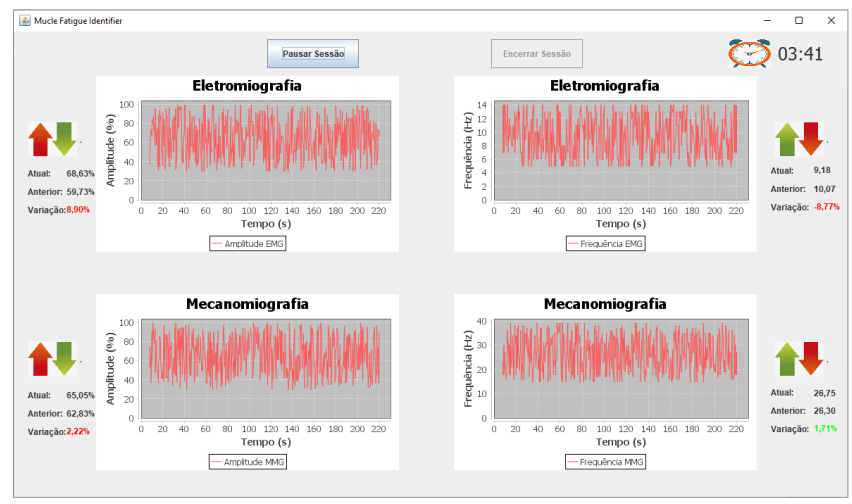

Figura 3: Tela de monitoramento da sessão.

Na tela de monitoramento, são exibidos ao usuário, em tempo de execução, quatro gráficos referentes aos sinais EMG e MMG. Além disso, o sistema realiza uma média parcial a cada 30 segundos e apresenta indicadores sobre a última e a atual parcial obtida, bem como um terceiro indicador que é a diferença entre as duas parciais. $\mathrm{O}$ indicador de variação apresenta cores que auxiliam o fisioterapeuta na interpretação dos dados, uma variação que indique que pode estar ocorrendo o processo de fadiga muscular será vermelha, enquanto uma variação que indique que não está ocorrendo o processo de fadiga muscular será verde. Como também é possível observar, existe um cronômetro para medir o tempo da sessão, assim como também dois botões para gerenciar a sessão, possibilitando ao fisioterapeuta as ações de iniciar, pausar, retomar ou encerrar a atividade no momento que desejar.

Após o término da sessão, o usuário é redirecionado a tela de relatórios, onde são apresentados os dados da sessão atual e, também, os registros das sessões anteriores, como demonstra a Figura 4.

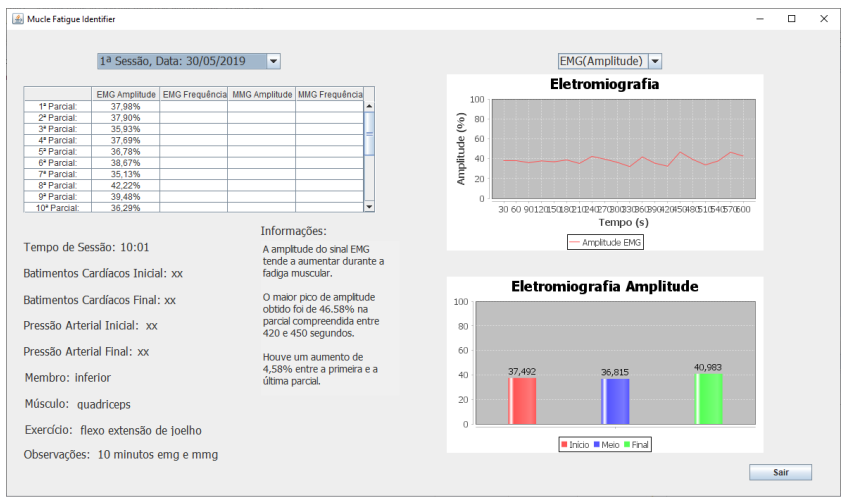

Figura 4: Tela de relatórios.

Nesta tela, se observa que no canto superior esquerdo é possível escolher a sessão que se deseja visualizar e no canto superior direito é possível escolher qual gráfico se deseja analisar (EMG Amplitude, EMG Frequência, MMG Amplitude ou MMG Frequência). Na tela são apresentados dois gráficos, o primeiro localizado no canto superior direito apresenta um gráfico de linha com as parciais obtidas durante a sessão, e o segundo, localizado no canto inferior direito, apresenta um gráfico de barra que faz um comparativo entre a média de três momentos da sessão (início, meio e final). Além dos dois gráficos, a tela de relatórios também apresenta uma tabela que contém todas as parciais obtidas para os quatro parâmetros durante as sessões.

Desse modo, o software desenvolvido recebe os dados do nó sensor, apresenta os resultados das coletas, gera indicadores, cadastra pacientes e armazena todas as informações no banco de dados, gerando um histórico completo de sessões de cada paciente.

\section{EXPERIMENTOS E VALIDAÇÃO}

Nesta seção serão apresentados os testes realizados para a validação da ferramenta, desde os testes unitários dos sensores até os experimentos com os pacientes do SRF. Desse modo, para a validação do sistema desenvolvido, foram elaborados testes que tiveram como objetivo avaliar o comportamento e o funcionamento de cada unidade que abrange a solução, como os sensores, a coleta de dados, o processamento de sinais, a comunicação serial, o software, a integração do sistema e, por fim, a validação. Assim, a Figura 5 ilustra, através de um diagrama, a sequência de etapas da realização de testes. 


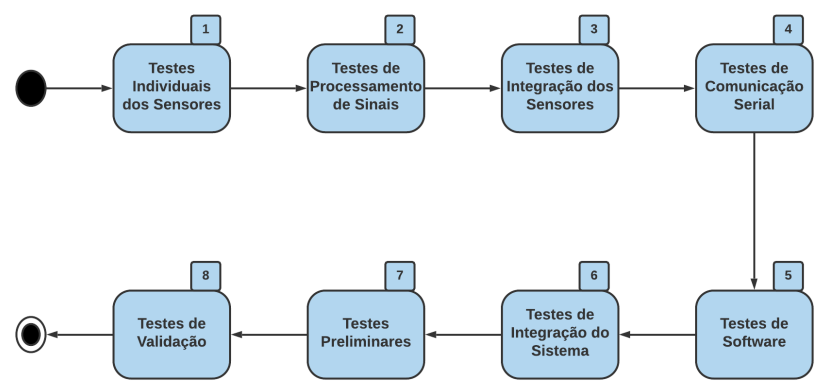

Figura 5: Diagrama de atividades representando a sequência de etapas da realização de testes.

A última etapa da realização de testes foi a de validação da solução apresentada neste trabalho. Desse modo, a validação foi feita nas instalações do SRF, com três pacientes que frequentam o serviço prestado na unidade de reabilitação física. Assim, os protocolos de atividades adotados durante a realização dos exercícios, bem como os pacientes que participaram das sessões, foram definidos pelos próprios fisioterapeutas, que acompanharam todas as etapas das avaliações. Desta maneira, a Tabela 1 apresenta as características dos pacientes que participaram da validação da solução.

Tabela 1: Pacientes selecionados para participação nos experimentos

\begin{tabular}{cccc}
\hline & Paciente 1 & Paciente 2 & Paciente 3 \\
\hline Sexo & Mulher & Homem & Homem \\
Idade & 84 & 37 & 72 \\
Nível Amputação & Transfemural & Transtibial & Transfemural \\
Lado Amputação & Esquerdo & Esquerdo & Esquerdo \\
\hline
\end{tabular}

A Tabela 2 apresenta as características das sessões realizadas pelos pacientes, sendo possível observar o exercício físico realizado, membro e músculo analisado, o tempo de duração da sessão e o paciente que realizou a sessão. Vale ressaltar que em relação ao tempo de duração dos experimentos, os fisioterapeutas optaram por realizar uma sessão livre, ou seja, sem tempo predeterminado, transcorrendo a atividade até o momento de desgaste dos pacientes.

Tabela 2: Características das sessões realizadas

\begin{tabular}{ccccc}
\hline & Sessão 1 & Sessão 2 & Sessão 3 & Sessão 4 \\
\hline Paciente & 1 & 2 & 2 & 3 \\
Membro & Inferior & Inferior & Inferior & Inferior \\
Músculo & Quadríceps & Quadríceps & Quadríceps & Quadríceps \\
Exercício & Ext.Joelho & Ext.Quadril & Ext.Joelho & Ext.Quadril \\
Duração & 10 minutos & 8 minutos & 3 minutos & 3 minutos \\
\hline
\end{tabular}

A Figura 6 apresenta a realização dos testes com os três pacientes do grupo experimental, onde se percebe a disposição dos sensores e, também, os exercícios realizados durante as sessões. O lado escolhido para o posicionamento dos sensores foi definido pelos fisioterapeutas, onde em três dos quatro experimentos a posição adotada foi no lado oposto à amputação e somente em um dos experimentos os sensores foram posicionados no lado da amputação.
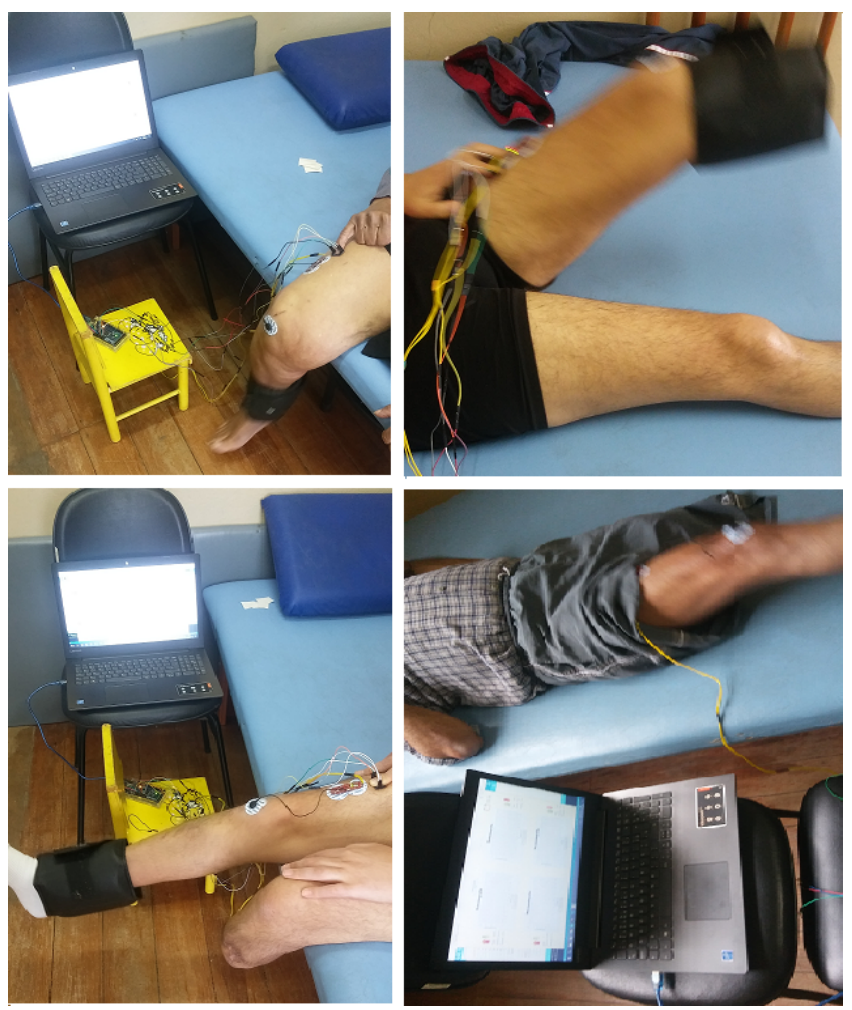

Figura 6: Realização de testes com pacientes.

Além da análise de fadiga muscular realizada pelo sistema desenvolvido, foi realizado um levantamento empírico sobre a condição física dos indivíduos durante as sessões. A cada minuto transcorrido de exercício o indivíduo descrevia numa escala de 1 a 5 o seu sentimento sobre o cansaço muscular, sendo 1 representando nenhum cansaço ou desconforto muscular e 5 representando um cansaço ou desconforto muscular extremo. Assim sendo, na próxima seção serão apresentados e discutidos os resultados obtidos durante a realização dos testes.

\section{RESULTADOS E DISCUSSÕES}

Nesta seção serão analisados e discutidos os resultados obtidos durante a etapa de validação da solução desenvolvida. Foram analisados os parâmetros de amplitude e frequência do sinal eletromiográfico, como também de amplitude e frequência do sinal mecanomiográfico, com o objetivo de validar a solução como um potencial identificador de fadiga muscular durante as sessões de fisioterapia.

Desta maneira, a Figura 7 apresenta os resultados obtidos para os parâmetros de amplitude e frequência dos sinais de eletromiografia e mecanomiografia durante as sessões realizadas pelos pacientes. É possível observar na Figura 7a que em todas as sessões o comportamento para a amplitude dos sinais foi semelhante, com o aumento deste parâmetro no final da atividade. Quanto à frequência dos sinais na Figura $7 \mathrm{~b}$, o comportamento também foi consistente, com a redução do parâmetro ao final da atividade. 


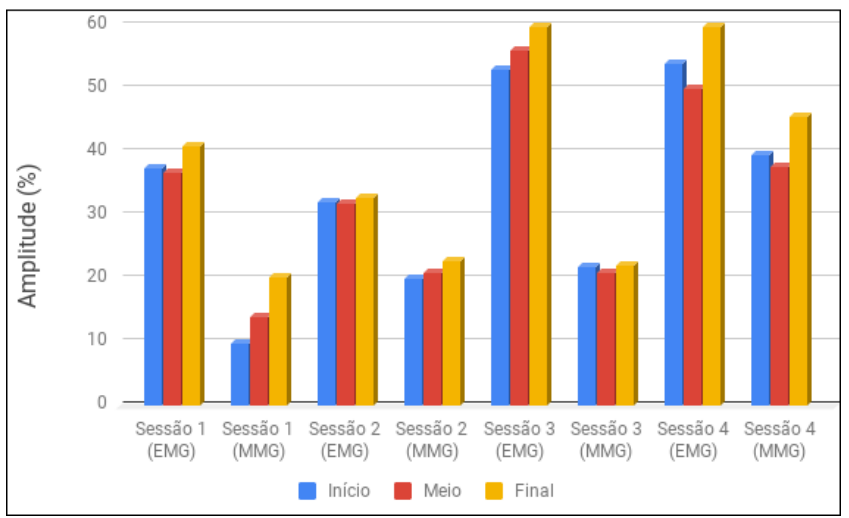

(a) Amplitude

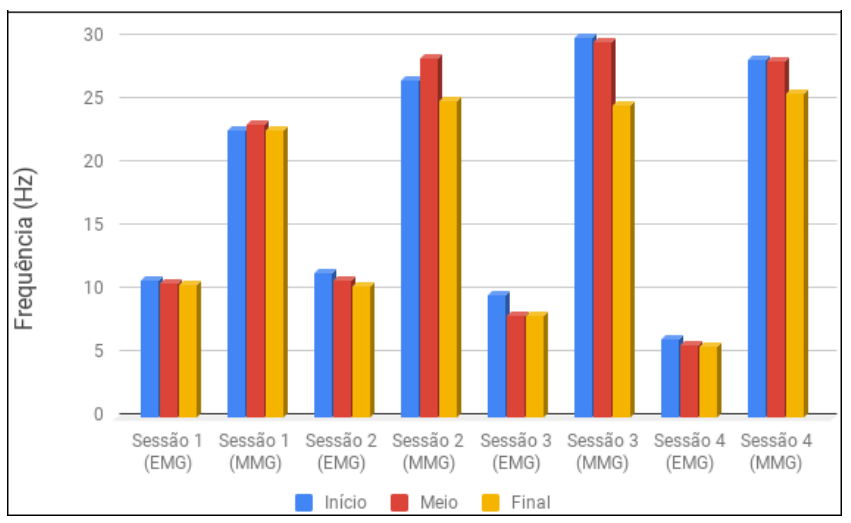

(b) Frequência

Figura 7: Resultados obtidos nos testes de validação do sistema.

Os resultados obtidos durante os testes de validação apontam para uma conformidade com os relatos encontrados na literatura, onde é considerado que durante a instauração da fadiga muscular ocorre um aumento na amplitude e uma redução na frequência dos sinais de eletromiografia e mecanomiografia.

Em seus estudos, [26] declara que os sinais de EMG e MMG registrados simultaneamente de um mesmo músculo tendem a possuir um comportamento similar, tanto para a EMG quanto para a MMG a frequência média diminui enquanto o valor da RMS da amplitude aumenta com a fadiga muscular. Seguindo este pensamento, [29] apontam que a fadiga está associada a uma compressão do PSD da EMG em direção à frequências menores, ao mesmo tempo que também está associada a amplitudes maiores do sinal.

Para a amplitude do sinal mecanomiográfico, a teoria mais aceita atualmente é do aumento gradativo da amplitude antes e após a instauração da fadiga muscular [12, 26].

Para uma melhor análise do grau de fadiga muscular identificado, como também para uma validação dos resultados apresentados pelo sistema MFID, se fez necessário uma comparação destes resultados com o nível de fadiga relatado pelos pacientes durante a realização das sessões. Para tanto, foi realizado o cálculo demonstrado na
Equação 2, o qual foi elaborado seguindo o entendimento e o consenso encontrados na literatura, onde a uma variação positiva na amplitude, como também uma variação negativa na frequência dos sinais de EMG e MMG indicam a instauração da fadiga muscular.

$$
\text { fadiga }=v_{a r_{\text {amp }}}-\text { var }_{\text {freq }_{E M G}}+\text { var }_{a m p_{M M G}}-\text { var }_{\text {freq }_{M M G}}
$$

Assim sendo, a Figura 8 apresenta esta comparação, onde o gráfico apresentado na parte superior demonstra o nível de fadiga informado pelos pacientes durante a realização das sessões, e o gráfico apresentado na parte inferior apresenta o valor identificado pelo sistema MFID para a fadiga muscular.

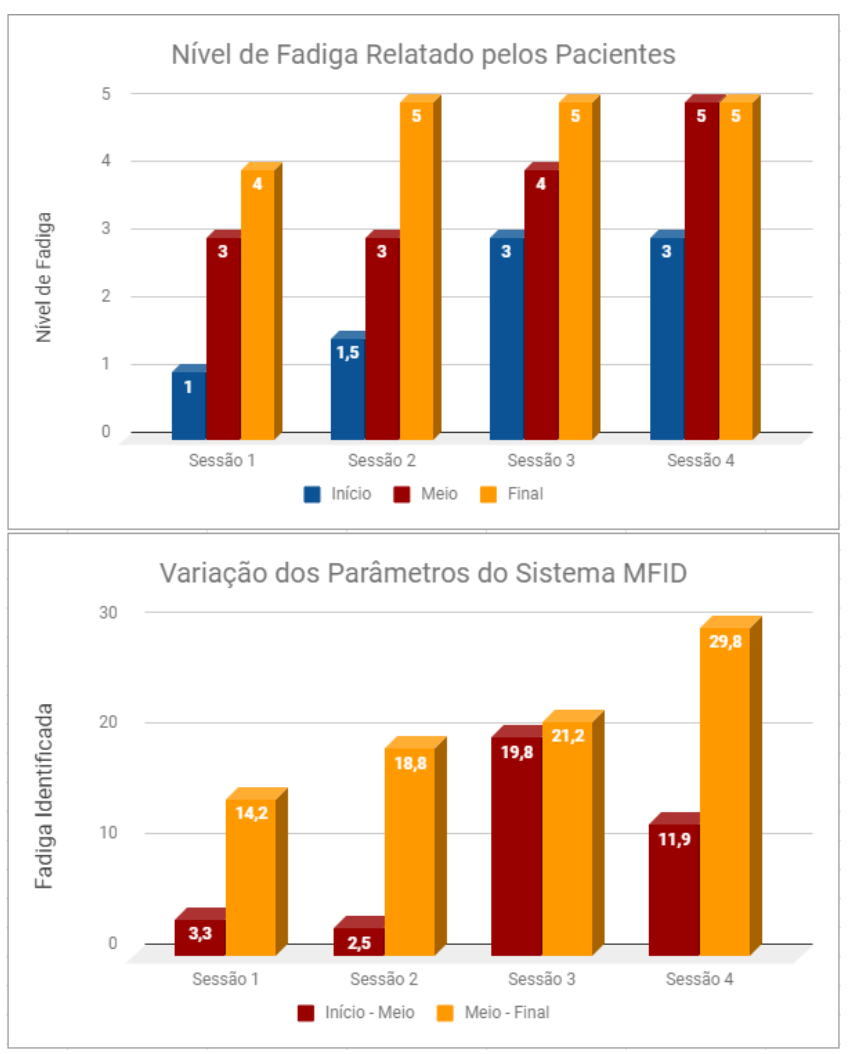

Figura 8: Comparação entre o nível de fadiga relatado pelos pacientes e o identificado pelo sistema MFID.

Ao analisar a figura, é possível observar uma concordância entre o nível fadiga indicada pelos pacientes e a fadiga identificada pelo sistema, pois foi a partir da metade das sessões onde foi informado pelos pacientes um elevado grau de cansaço e desconforto muscular, e ao observar os valores encontrados pelo sistema, é entre o meio e o final das sessões onde foi identificada a maior variação dos parâmetros que indicam o surgimento da fadiga muscular.

No geral, ao analisar todos os resultados obtidos pelo sistema MFID percebe-se que as médias dos três estágios das atividades (início, meio e final) apresentaram um comportamento consistente para os dois sinais estudados, ou seja, um aumento no valor da amplitude ao mesmo tempo em que a frequência reduzia. Em todas as sessões os indivíduos demonstraram sinais de fadiga muscular 
e, de acordo com a literatura, os resultados encontrados apontam para a ocorrência deste fenômeno.

Analisando os parâmetros utilizados no estudo da fadiga muscular, foi possível observar que o sinal de EMG apresentou melhores resultados quanto à variação das parciais se comparado ao sinal MMG. Também é possível considerar que a amplitude dos sinais apresentou melhor consistência em seus resultados do que a frequência. Contudo, ainda são necessárias mais avaliações e análises sobre o comportamento destes parâmetros para uma consolidação da pesquisa.

\section{CONCLUSÃO}

O objetivo deste trabalho foi desenvolver uma solução computacional capaz de auxiliar o profissional fisioterapeuta, através do monitoramento e identificação da fadiga muscular, durante o processo de reabilitação física de pacientes amputados. Desta forma, a relevância deste trabalho se comprova pela a atual realidade do processo de reabilitação física de pacientes do SRF, onde a avaliação de cada sessão, como também de todo o progresso dos pacientes ao longo do tratamento não possui um aparato tecnológico que auxilie na análise física do paciente, bem como da evolução do tratamento.

É importante avaliar a opinião dos fisioterapeutas sobre os resultados obtidos, bem como de todo o sistema desenvolvido. Durante as reuniões e experimentos realizados, foi relatada a relevância da pesquisa para o aprimoramento do tratamento, como também o feedback positivo ao analisar o funcionamento e os resultados apresentados pelo sistema. Desse modo, é possível considerar que o projeto MFID alcançou os objetivos de pesquisa.

Portanto, a partir da utilização do sistema MFID é possível obter um aperfeiçoamento no método de acompanhamento e avaliação do tratamento de pacientes, uma vez que o sistema proporciona aos fisioterapeutas informações quantitativas sobre as sessões, facilitando o diagnóstico, adaptação e particularização do tratamento conforme as individualidades de cada caso.

Além dos resultados obtidos, é relevante ressaltar o carácter social da pesquisa. O levantamento de referencial teórico oportunizou um melhor entendimento sobre o cenário atual da reabilitação física, bem como sobre os aspectos relacionados à amputação. Assim, a pesquisa contribuiu não só na formação acadêmica, mas também na formação social e humana, visto que a pesquisa tem como objetivo aprimorar o processo de reabilitação física de pacientes amputados.

Por fim, pretende-se dar continuidade no desenvolvimento do sistema MFID a partir da realização de novos testes com pacientes do Serviço de Reabilitação Física e a integração deste sistema com outros sistemas voltados à reabilitação física de pacientes amputados desenvolvidos dentro do Grupo de Informática Médica. Além disso, visando um aperfeiçoamento no sistema, é analisada a utilização de ferramentas e técnicas mais sofisticadas para a coleta e processamento de sinais, como também a adição de mais sensores para ampliar e aprimorar o monitoramento das sessões.

\section{REFERENCES}

[1] Christine Detrembleur, Jean-Marie Vanmarsenille, Freddy De Cuyper, and Frédéric Dierick. Relationship between energy cost, gait speed, vertical displacement of centre of body mass and efficiency of pendulum-like mechanism in unilateral amputee gait. Gait \& posture, 21:333-340, April 2005. ISSN 0966-6362. doi: 10.1016/j.gaitpost.2004.04.005.
[2] A. V. Sampol. Tratamento fisioterápico no amputado de membro inferior no periodo ambulatorial. Nova Fisio. Vol. 2, 2000.

[3] Antonio Carlos Gil. Métodos e técnicas de pesquisa social. 6. ed. Ediitora Atlas SA, 2008.

[4] Tatiana Engel Gerhardt and Denise Tolfo Silveira. Métodos de pesquisa. Plageder, 2009.

[5] J. Hawley and A. Reilly. Fatigue revisited. fournal of Sports Sciences, 1997.

[6] António Ascensão, José Magalhães, José Oliveira, JA Duarte, and José Soares. Fisiologia da fadiga muscular. delimitação conceptual, modelos de estudo e mecanismos de fadiga de origem central e periférica. 2003.

[7] Jean-Louis Croisier, Benoît Maertens De Noordhout, Didier Maquet, Gérard Camus, S Hac, Frédéric Feron, O De Lamotte, and Jean-Michel Crielaard. Isokinetic evaluation of hip strength muscle groups in unilateral lower limb amputees. Isokinetics and exercise science, 9(4):163-169, 2001.

[8] Mario A Lafortune, Ewald Henning, and Gordon A Valiant. Tibial shock measured with bone and skin mounted transducers. Fournal of biomechanics, 28(8):989-993, 1995.

[9] L Portney and SH Roy. Eletromiografia e testes de velocidade de condução nervosa. Fisioterapia: Avaliação e Tratamento. São Paulo: Manole, pages 183-217, 1993.

[10] C Russell Hendrix, Terry J Housh, Glen O Johnson, Michelle Mielke, Clayton L Camic, Jorge M Zuniga, and Richard J Schmidt. A new emg frequency-based fatigue threshold test. Fournal of neuroscience methods, 181(1):45-51, 2009.

[11] Kumi Akataki, Katsumi Mita, Makoto Watakabe, and Kunihiko Itoh. Mechanomyographic responses during voluntary ramp contractions of the human first dorsal interosseous muscle. European journal of applied physiology, 89 (6):520-525, 2003.

[12] Yasuhide Yoshitake, Minoru Shinohara, Hidetoshi Ue, and Toshio Moritani. Characteristics of surface mechanomyogram are dependent on development of fusion of motor units in humans. Fournal of Applied Physiology, 93(5):1744-1752, 2002.

[13] Ana Patrícia Matos da Costa Silva. Amputação e reabilitação: Estudo dos aspectos psico-legais do sujeito com amputação. $\mathrm{PhD}$ thesis, Instituto Superior de Psicologia Aplicada, 2006.

[14] Sérgio Lianza. Medicina de reabilitação. In Medicina de reabilitação. 1995.

[15] D Lavin, D Hevey, HM McGee, D De la Harpe, M Kiernan, and E Shelley. Cardiac rehabilitation services in ireland: the impact of a coordinated national development strategy. Irish journal of medical science, 174(4):33-38, 2005.

[16] Tanneke Schoppen, Annemarijke Boonstra, Johan W Groothoff, Jaap de Vries, Ludwig N Göeken, and Willem H Eisma. Physical, mental, and social predictors of functional outcome in unilateral lower-limb amputees1. Archives of physical medicine and rehabilitation, 84(6):803-811, 2003.

[17] P. B. Schweitzer. Fisioterapia ortopédica e medicina ortopédica. Fisioter Bras, 5 (5):375-379, 2004

[18] Fahim Anwar and Ahmad Alkhayer. Perceptions of prosthetic limb among lower limb amputees. International fournal of Therapies and Rehabilitation Research, 5 (4):175-179, 2016

[19] JC Wyatt and JLY Liu. Basic concepts in medical informatics. Fournal of Epidemiology \& Community Health, 56(11):808-812, 2002.

[20] Enrico Coiera. Guide to health informatics. CRC press, 2015.

[21] Jefferson Paixão Cardoso. O uso de sistemas especialistas para apoio à sistematização em exames ortopédicos do quadril, joelho e tornozelo. Revista Saude. com, 1(1):24-34, 2016.

[22] Robert I Levine. Inteligência artificial e sistemas especialistas. McGraw-Hill, 1988.

[23] Nicolai Diniz Linhares. Análise dos efeitos da fadiga muscular no sinal eletromiográfico de superfície em contrações dinâmicas do bíceps braquial. 2015.

[24] Bruno Jotta da Costa. Caracterização do sinal mecanomiográfico através da acelerometria em três diferentes músculos. $\mathrm{PhD}$ thesis, Universidade Federeal do Rio de Janeiro, 2008.

[25] Lilian Faller. Mecanomiografia como técnica de detecção de fadiga muscular durante a aplicação da estimulação elétrica neuromuscular (EENM). 2007.

[26] Mihai T Tarata. Mechanomyography versus electromyography, in monitoring the muscular fatigue. Biomedical engineering online, 2(1):3, 2003.

[27] Roger M Enoka and Jacques Duchateau. Muscle fatigue: what, why and how it influences muscle function. The fournal of physiology, 586(1):11-23, 2008.

[28] Travis W Beck, Terry J Housh, Glen O Johnson, Joseph P Weir, Joel T Cramer, Jared W Coburn, and Moh H Malek. Mechanomyographic amplitude and mean power frequency versus torque relationships during isokinetic and isometric muscle actions of the biceps brachii. Fournal of Electromyography and Kinesiology, 14(5):555-564, 2004.

[29] Foster B Stulen and Carlo J De Luca. Frequency parameters of the myoelectric signal as a measure of muscle conduction velocity. IEEE Transactions on Biomedical Engineering, (7):515-523, 1981. 